\title{
BubR1 Insufficiency Impairs Affective Behavior and Memory Function in Mice
}

\author{
Chang Hoon Cho ${ }^{1, \star}$, Zhongxi Yang ${ }^{1,3, *}$, Ki Hyun Yoo $^{1}$, Alfredo Oliveros ${ }^{1}$, Mi Hyeon Jang ${ }^{1,2}$ \\ ${ }^{1}$ Department of Neurologic Surgery, Mayo Clinic College of Medicine, Rochester, MN, USA \\ ${ }^{2}$ Department of Biochemistry and Molecular Biology, Mayo Clinic College of Medicine, Rochester, MN, USA \\ ${ }^{3}$ Department of Neurologic Surgery, First Hospital of Jilin University, Changchun, China
}

Purpose: Although aging causes functional declines in cognition, the molecular mechanism underlying these declines remains largely unknown. Recently, the spindle checkpoint kinase budding uninhibited by benzimidazole-related 1 (BubR1) has emerged as a key determinant for age-related pathology in various tissues including brain. However, the neurobehavioral impact of BubR1 has not been explored. In this study, we investigated the role of BubR1 in behavioral function.

Methods: To investigate the neurobiological functions of BubR1 in vivo, we utilized transgenic mice harboring BubR1 hypomorphic alleles ( $B u b R 1^{\mathrm{H} / \mathrm{H}}$ mice), which produce low amounts of BubR1 protein, as well as mice that have specific knockdown of BubR1 in the adult dentate gyrus. To assess anxiety-like behavior, the above groups were subjected to the elevated plus maze and the light-dark test, in addition to utilizing the tail-suspension and forced-swim test to determine depression-like behavior. We used novel object recognition to test for memory-related function.

Results: We found that $B u b R 1^{\mathrm{H} / \mathrm{H}}$ mice display several behavioral deficits when compared to wild-type littermates, including increased anxiety in the elevated-plus maze test, depression-like behavior in the tail suspension test, as well as impaired memory function in the novel object recognition test. Similar to BubR $1^{\mathrm{H} / \mathrm{H}}$ mice, knockdown of BubR1 within the adult dentate gyrus led to increased anxiety-like behavior as well as depression-like behavior, and impaired memory function.

Conclusions: Our study demonstrates a requirement of BubR1 in maintaining proper affective and memory-related behavioral function. These results suggest that a decline in BubR1 levels with advanced age may be a crucial contributor to age-related hippocampal dysfunction.

Keywords: BubR1; Aging; Hippocampus; Memory; Emotion

- Fund/Grant Support: This work was supported by NIA (R01AG058560), Regenerative Medicine Minnesota, Mayo Clinic Center for Regenerative Medicine, and Department of Neurologic Surgery at Mayo Clinic to MHJ, and post-doctoral fellowship from Center for Regenerative Medicine at Mayo Clinic to KHY.

- Research Ethics: Animal care and handling procedures were approved by the Mayo Clinic Institutional Animal Care and Use Committee (IA-

CUC; \#A1756) in accordance with National Institutes of Health guidelines.

- Conflict of Interest: No potential conflict of interest relevant to this article was reported.

\section{- HIGHLIGHTS}

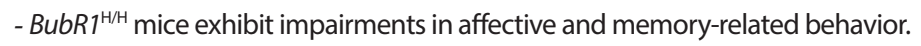

- Dentate gyrus-specific BubR1 knockdown mice exhibit impairments in affective and memory-related behavior.

- Dentate gyrus-specific BubR1 knockdown causes neurogenic dysfunction in a non-cell autonomous manner.

Corresponding author: Mi Hyeon Jang (iD http://orcid.org/0000-0001-6274-3588 Department of Neurologic Surgery, Mayo Clinic College of Medicine, 200 First Street SW, Rochester, MN 55905, USA

E-mail: jang.mihyeon@mayo.edu / Tel: +1-507-284-1219 / Fax: +1-507-284-3383

${ }^{*}$ Chang Hoon Cho and Zhongxi Yang contributed equally to this study as co-first authors.

Submitted: September 16, 2018 / Accepted after revision: October 12, 2018
This is an Open Access article distributed under the terms of the Crecc)
commons.org/licenses/by-nc/4.0/) which permits unrestricted non-commercial use, distribution, and reproduction in any medium, provided the original work is properly cited. 


\section{INTRODUCTION}

Aging is the greatest risk factor for cognitive dysfunction and neurodegenerative disorders [1]. It is estimated that the global population advancing in age will increase over the next several decades [2], making the incidence of age-related cognitive decline a significant concern for aging individuals. Despite the high prevalence of aging and age-related disorders, present therapeutic development is impeded by inadequate understanding of the cellular and molecular mechanisms that contribute to cognitive decline in aging individuals.

The spindle assembly checkpoint kinase known as budding uninhibited by benzimidazole-related 1 (BubR1) has emerged as a key genetic component for aging and age-related pathology $[3,4]$. BubR1 is a multifunctional serine/threonine kinase necessary for appropriate chromosome segregation, kinetochore-microtubule attachment, and timing of mitosis through the APC/ $\mathrm{C}^{\mathrm{Cd} 220}$ signaling pathway [5]. Notably, expression levels of BubR1 in wild-type (WT) mice gradually decline over the aging process in multiple tissues including the brain. Transgenic mice harboring BubR1 hypomorphic alleles ( $B u b R 1^{\mathrm{H} / \mathrm{H}}$ mice) producing low amounts of BubR1 develop an accelerated aging phenotype that includes a short lifespan, cataracts, cachectic dwarfism, and sarcopenia [3]. In humans, mutations in the BUB1B gene that encodes the BubR1 protein are linked to mosaic variegated aneuploidy (MVA) syndrome [6]. Similar to phenotypes observed in $B u b R 1^{\mathrm{H} / \mathrm{H}}$ mice, patients with MVA syndrome express low levels of BubR1 and exhibit progeria traits such as short lifespans, diminished stature, facial dysmorphisms, and cataracts [7-9]. Notably, children with MVA syndrome also exhibit microcephaly, central nervous system abnormalities, seizures, and defects in ciliogenesis [6,9-14], implying a critical role of BubR1 in brain development and function. In our previous reports, we demonstrated that BubR1 insufficiency impairs hippocampal neurogenesis [15] and myelination [16], both of which are associated with age-related neuropathology contributing to cognitive dysfunction. Although these studies strongly suggest a crucial role for BubR1 in brain development and aging-related pathways, it is currently unknown whether declines in BubR1 also contribute to cognitive function and affective responses.

In the present study, we investigated the role of BubR1 in behavioral function. Using $B u b R 1^{\mathrm{H} / \mathrm{H}}$ mice which prematurely age due to deficits in BubR1 levels, and mice with a lentivirus-mediated shRNA BubR1 knockdown, we sought to test the hypothesis that a reduced level of BubR1 impairs memory related cognitive function as well as affective responses including anxiety and depressive-like behavior.

\section{MATERIALS AND METHODS}

\section{Mice}

$B u b R 1^{\mathrm{H} / \mathrm{H}}$ mice was a generous gift provided by Dr. Jan M. van Deursen at Mayo Clinic. BubR ${ }^{\mathrm{H} / \mathrm{H}}$ mice were generated as described previously [3,4], and were backcrossed to the C57BL/6 background for over 10 generations. They were housed in standard cages under a 12-hour light/dark cycle. Standard rodent chow and water were provided ad libitum. Animal care and handling procedures were approved by the Mayo Clinic Institutional Animal Care and Use Committee (IACUC; \#A1756) in accordance with National Institutes of Health guidelines.

\section{Behavioral Analysis}

All behavior tests were performed in the rodent behavior facility of the Mayo Clinic College of Medicine. Adult male $B u b R 1^{\mathrm{H} / \mathrm{H}}$ and their WT littermates at the age of 8-10 weeks, and BubR1 knockdown or control mice at the age of 12-14 weeks were used at the time of behavior performed. Mice were transferred from the housing room to the behavior facility holding area 1 hour before testing. All tests were performed during the dark phase of the 12-hour light/dark cycle. Testing chambers were sanitized with $70 \%$ ethanol between trials. Unless otherwise noted, mouse movement was recorded by a video webcam (HDR-CX260, Sony, Tokyo, Japan) and analyzed by off-line video tracking software (EthoVision XT 10, Noldus Information Technology, Leesburg, VA, USA). At the end of all experiments, mice were returned to their home cage and transferred back to the holding room.

\section{Elevated plus maze}

Elevated plus maze (EPM) test apparatus consisted of 2 open arms (length $32 \times$ width $10 \mathrm{~cm}$ ) and 2 closed arms of the same size, along with a semi-transparent wall (height $26 \mathrm{~cm}$ ) and central platform (length $10 \times$ width $10 \mathrm{~cm}$ ). These arms and central platform were elevated $50 \mathrm{~cm}$ above the floor. Each mouse was placed in the edge of the open arm facing the closed arms and recorded using EthoVision XT (EthoVision XT 10, Noldus Information Technology, Leesburg, VA, USA) for 5 minutes. The number of entries into the open and closed arms, as well as the time spent in the open and closed arms were measured as previously described with slight modification [17]. 


\section{Tail suspension test}

The tail suspension box was customized and each chamber (55 $\mathrm{cm} \times 15 \mathrm{~cm} \times 11 \mathrm{~cm}$ ) was separated from the other chambers by a partition to prevent animals from interacting with each other. The approximate distance between the mouse's nose and the bottom of the suspension box is $20-25 \mathrm{~cm}$. Mice were suspended by the tail by gently securing the tail with sticky tape on a paper clip that was then attached to a length of string. The total time spent immobile was recorded over a 6-minute period [18].

\section{Forced swimming test}

Mice were gently placed in a glass cylinder $(18 \mathrm{~cm}$ in diameter) filled with $10 \mathrm{~cm}$ of water $\left(25^{\circ} \mathrm{C}-26^{\circ} \mathrm{C}\right)$ to prevent mice from touching the bottom of the cylinder with their paws or tail. The amount of time spent immobile was recorded across a period of 6 minutes. Immobility during the last 4 minutes of the 6-minute trial was measured with the aid of EthoVision XT (Noldus Information Technology, Leesburg, VA, USA) as we used previously [18]. After testing, each mouse was placed in a warm holding cage $\left(30^{\circ} \mathrm{C}-35^{\circ} \mathrm{C}\right)$ with bedding covered by a paper towel.

\section{Light-dark test}

The apparatus was a rectangular box made of Plexiglas divided by a partition into 2 compartments. One compartment was dark and the other compartment was brightly illuminated. The time spent in the dark compartment was recorded and each session had a 5-minute duration [19].

\section{Novel objective recognition test}

The novel object recognition task is conducted in an open field arena with 2 different kinds of objects. Both objects are generally consistent in height and volume, but are different in shape and appearance. Mice were habituated to the empty arena for 30 minutes on day 1 . On familiarization day 2 , individual mice were introduced to the same arena and exposed to 2 identical objects (F1 and F2) for 10 minutes. On test day 3, the mice were again placed in the arena, however in addition to the familiar object 1 (F1), a novel object (N) was introduced to test longterm recognition memory. A camera mounted above the arena recorded the animal's general behavior and was analyzed via Ethovision XT tracking software (Noldus Information Technology, Leesburg, VA, USA). Object interaction was also scored by an experimenter blinded to the control and experimental groups tested. The total time spent exploring the familiar and novel object is recorded [20].

\section{Construction and Lentiviral Production}

The short-hairpin sequences used are as follows: (shRNA-BubR1 \#1) 5'-AAGGGAAGCCGAGCTGTTGAC-3'; (shRNABubR1 \#2) 5'- AGATCCTGGCTAACTGTTC-3'. Knockdown efficiency of B\#1 and B\#2 constructs was approximately 70\%$75 \%$ in vitro [15]. Highly concentrated lentiviruses coexpressing shRNA-BubR1 (B\#1 and B\#2) or shRNA-control and green fluorescence protein (GFP) were stereotaxically injected into the dentate gyrus (DG) of adult C57BL/6 WT mice. Adult male C57BL/6 mice at 7-8 weeks of age housed under standard conditions were anaesthetized and lentiviruses were stereotaxically injected into the DG at 4 sites $(0.5 \mu \mathrm{L}$ per site at $0.25 \mu \mathrm{L} / \mathrm{min})$ with the following coordinates (in $\mathrm{mm}$ ): posterior $=2$ from Bregma, lateral $= \pm 1.6$, ventral $=2.5$; posterior $=3$ from Bregma, lateral $= \pm 2.6$, ventral $=3.2$ as described previously [21]. At 4 weeks postviral injection, mice were behavior tested.

\section{EdU Labeling, Immunohistochemistry, Confocal Imaging, and Analysis}

For cell proliferation analysis, adult male C57BL/6 mice at 8-10 weeks of age housed under standard conditions were anaesthetized and lentiviruses were stereotaxically injected into the DG at 4 sites as stated above. At 14 days postviral injection, mice were injected with 5-ethynyl-2'-deoxyuridine (EdU) (41.1-mg/ $\mathrm{kg}$ body weight, intraperitoneally) to label dividing cells, and transcardially perfused 2 hours later with cold phosphate buffered saline followed by $4 \%$ paraformaldehyde solution. After postfixation and cryoprotection, coronal brain sections $(40 \mu \mathrm{m})$ were made in serial order for a total of -50 sections along the anterior-posterior axis using a microtome (Leica SM 2010R; Leica Biosystems Inc., Buffalo Grove, IL, USA). Approximately 4-5 brain sections in each group were obtained in serial order of DG from the anterior to posterior axis, and processed for immunostaining as previously described [22]. EdU staining was performed according to manufacturer's instructions (Thermo Fisher Scientific, Waltham, MA, USA). Stereological quantification of EdU ${ }^{+}$cells in the subgranular zone (SGZ) were performed as previously described [15]. The volume of the DG section was calculated by multiplying the area by its thickness. The cell count was divided by the resulting section volume to obtain the total cell density in the DG per $\mathrm{mm}^{3}$.

For dendritic development analysis, adult male C57BL/6 mice at 8-10 weeks of age were anaesthetized and tdTomato-expressing lentiviruses were stereotaxically injected into the DG. At 14 days postviral injection, GFP-expressing retroviruses were 
stereotaxically injected with into the same sites 14 days after lentiviral (LV) injection and mice were subsequently examined following an additional 14 days for morphological analysis.

\section{Statistical Analysis}

Statistical analysis was performed using GraphPad Prism 7.05 (GraphPad Sofrware, La Jolla, CA, USA). Student t-test and 1-way analysis of variance (ANOVA) was performed with Bonferroni post hoc test for multiple comparisons. Statistical significance was defined as $\mathrm{P}<0.05$.

\section{RESULTS}

\section{BubR $1^{\mathrm{H} / \mathrm{H}}$ Mice Exhibit Increased Anxiety- and Depressive- Like Behavior with Impaired Memory Function}

Mutations of BubR1 are linked to intellectual disability [9]. Therefore, we investigated whether BubR1 can regulate emotional and memory-related behaviors. First, to test if BubR1 regulates emotional behaviors, $B u b R 1^{\mathrm{H} / \mathrm{H}}$ mice and WT littermate controls were assessed for anxiety with the EPM and light dark (LD) test. As depicted in Fig. 1A (left), we revealed that $B u b R 1^{\mathrm{H} / \mathrm{H}}$ mice spent significantly less time in the open arms of the EPM, while increasing the amount of time spent in the closed arms. Interestingly, we also found that the number of open arm entries were significantly lower when compared to WT controls (Fig. 1A, right). We then assessed anxiety with an alternative anxiety assay, the LD test, and found that relative to WT controls, $B u b R 1^{\mathrm{H} / \mathrm{H}}$ mice spent less time in the lighted, exposed compartment of the LD chamber (Fig. 1B). Although our $\mathrm{LD}$ test result did not reach statistical significance $(\mathrm{P}=0.07)$, when considered in conjunction with increased anxiety in the EPM, it suggests that BubR1 may play a role in regulation of anxiety. Next, we investigated the involvement of BubR1 in depressive-like behavior utilizing the tail suspension test, and found that $B u b R 1^{\mathrm{H} / \mathrm{H}}$ mice exhibited increased time spent immobile, suggesting a role for BubR1 insufficiency in depressive-
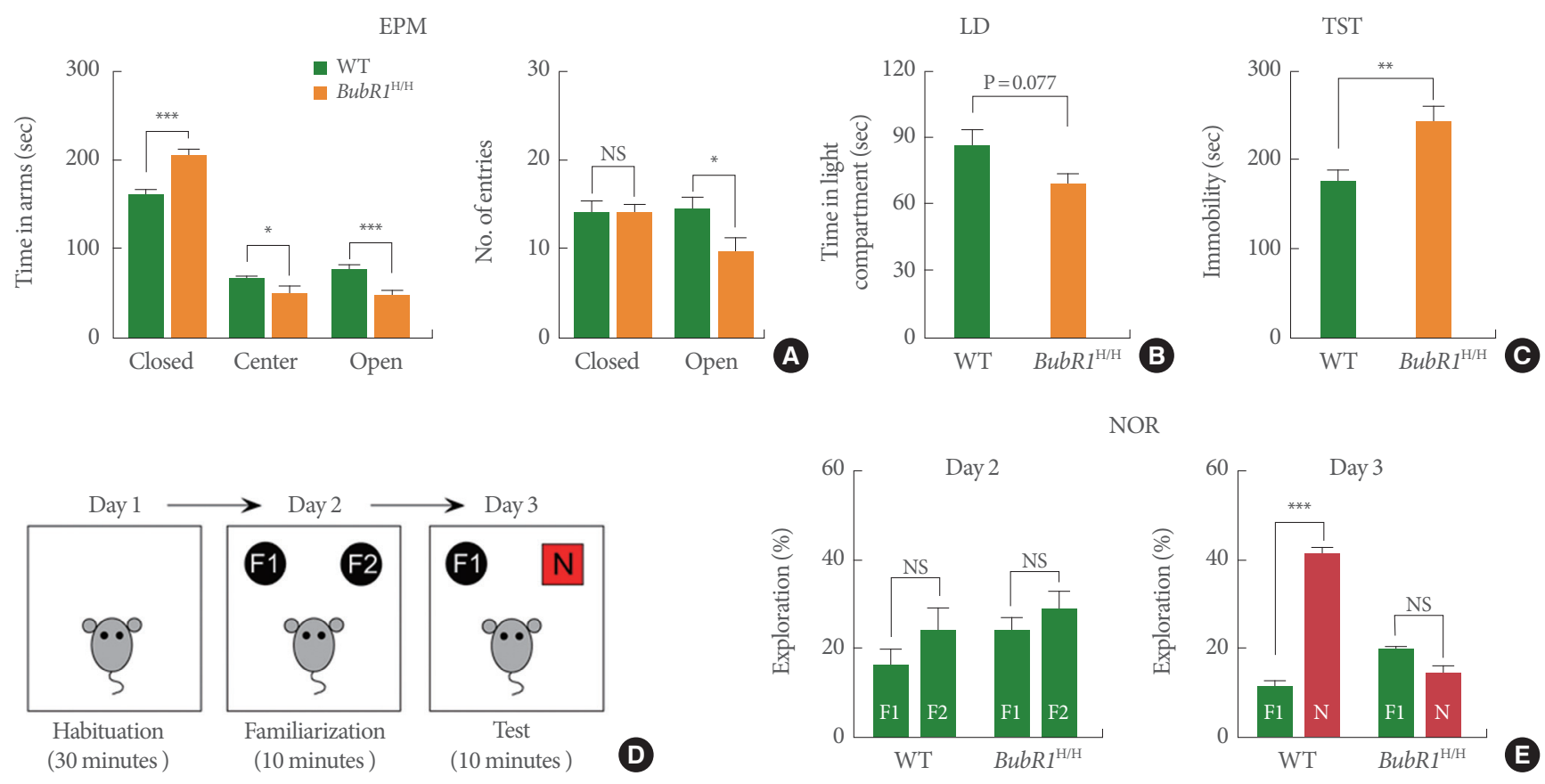

Fig. 1. Benzimidazole-related 1 hypomorphic $\left(B u b R 1^{\mathrm{H} / \mathrm{H}}\right)$ mice exhibit increased anxiety, depressive-like behaviors and impaired memory function. (A) Elevated plus maze (EPM) test: Left is a summary of time spent in close, center and open arms. Right is a summary of number of entries into open or closed arm. BubR1 ${ }^{\mathrm{H} / \mathrm{H}}$ mice spent increased time in the closed arms and a decreased time in the open arms relative to wild-type (WT) mice, suggesting increased anxiety in BubR ${ }^{\mathrm{H} / \mathrm{H}}$ mice. (B) Light-dark (LD) test. (C) Tail suspension test (TST): Bu$b R 1^{\mathrm{H} / \mathrm{H}}$ mice display increased immobility time. (D, E) Novel objective recognition (NOR) test: (D) Schematic diagram of NOR. (E) In the NOR familiarization phase on day 2, both $B u b R I^{\mathrm{H} / \mathrm{H}}$ and WT mice showed no differences in time spent exploring the 2 familiar objects, indicating a lack of location preference. In test phase day $3, B u b R 1^{\mathrm{H} / \mathrm{H}}$ mice spent less time exploring the novel object, relative to WT littermates, suggesting impaired recognition memory in $B u b R 1^{\mathrm{H} / \mathrm{H}}$ mice. All values represent mean \pm standard error of the mean $(17 \mathrm{BubR} 1 \mathrm{H} /$ $\mathrm{H}$ mice and 12 WT mice). F1, F2, familiar objects; $\mathrm{N}$, novel object; NS, nonsignificant. ${ }^{*} \mathrm{P}<0.05 .{ }^{* *} \mathrm{P}<0.01 .{ }^{* * *} \mathrm{P}<0.001$. Student t-test. 
like behavior (Fig. 1C). Taken together, our anxiety and depressive-like behavior findings suggest that deficits in expression of BubR1 may potentiate dysfunctional affective responses.

Next, to determine the involvement of BubR1 in recognition memory, we carried out the novel objective recognition (NOR) test (Fig. 1D), a well characterized assay known to be strongly affected by hippocampal impairment [23]. As displayed on Fig. $1 \mathrm{E}$, our results show that in the NOR familiarization phase (day 2), both WT and $B u b R 1^{\mathrm{H} / \mathrm{H}}$ mice did not display differences in time spent exploring the 2 familiar objects (F1 and F2), indicating a lack of location preference. However, introduction of the novel object (test phase, day 3), $B u b R 1^{\mathrm{H} / \mathrm{H}}$ mice spent a similar amount of time exploring the novel object $(\mathrm{N})$ and the familiar object (F1). This is in contrast with WT littermates, which as expected, spent significantly more time exploring the novel object $(\mathrm{N})$. Therefore, our findings strongly suggest that BubR1 insufficiency impairs recognition memory function.

\section{Dentate Gyrus-Specific BubR1 Knockdown Increases Anxiety, Depressive-Like Behavior and Impairs Memory Function}

Our previous research demonstrated that $B u b R 1^{\mathrm{H} / \mathrm{H}}$ mice develop significant motor and movement abnormalities at 2-3
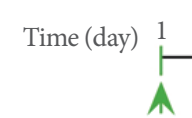

ShRNA-BubR1 LV

shRNA-control LV

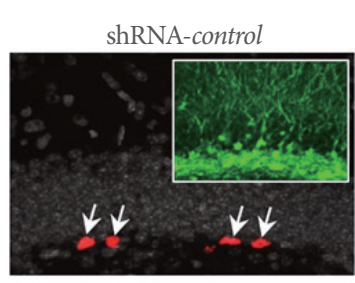

GFP-expressing lentivirus/EdU/DAPI

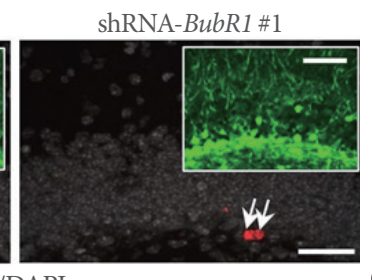

B

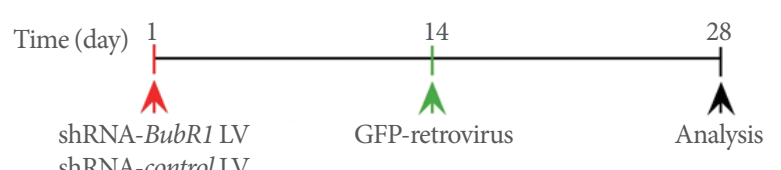

shRNA-control LV

shRNA-control
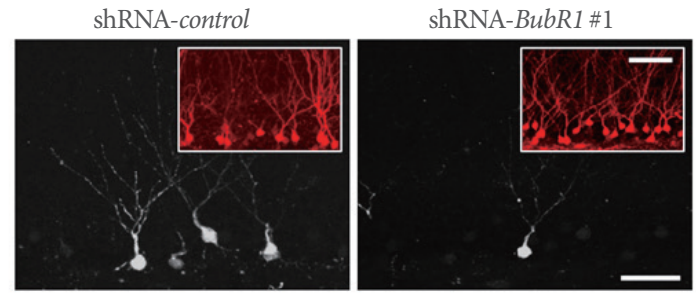

tdTomato-expressing lentivirus/GFP-expressing retrovirus
D

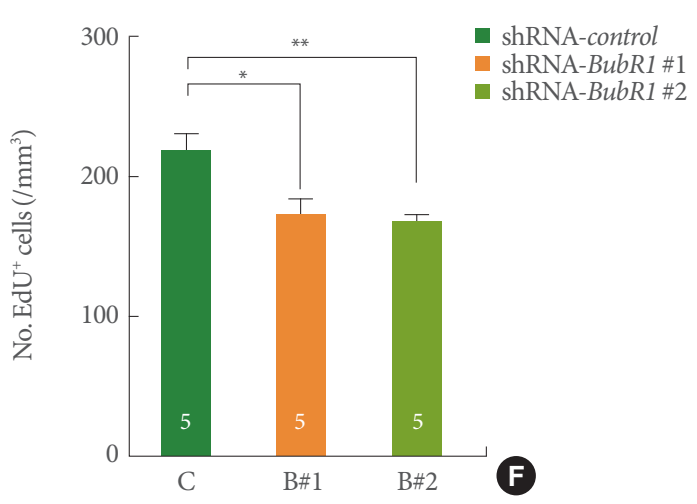

Fig. 2. Dentate gyrus (DG)-specific benzimidazole-related 1 (BubR1) knockdown impairs neural progenitor proliferation and dendrite morphogenesis of newborn neurons in a non-cell autonomous manner. (A-C) Neural progenitor proliferation: (A) Schematic diagram of experimental design. (B) Representative confocal images of green fluorescence protein (GFP)-expressing lentiviruses (green; inset), 5-ethynyl-2'-deoxyuridine (EdU, red), and 4', 6'-diamidino-2-phenylindole (DAPI, gray) in the adult mouse DG. Scale bars: $20 \mu \mathrm{m}$, respectively. (C) Stereological quantification of EdU ${ }^{+}$cells in the adult subgranular zone. (D-F) Dendrite morphogenesis analysis: (D) Schematic diagram of experimental design. (E) Representative confocal images of tdTomato-expressing lentiviruses (red; inset), and GFP-expressing retrovirus (white) in the adult mouse DG. Scale bars: $50 \mu \mathrm{m}$, respectively. (F) A summary of mean values of total dendritic length of newborn neurons. Decreased dendritic length using 2 different shRNA mediated BubR1 knockdown constructs, $\mathrm{B} \# 1$ and $\mathrm{B} \# 2$. All values represent mean \pm standard error of the mean. 1-way analysis of variance with Bonferroni post hoc test. ${ }^{*} \mathrm{P}<0.05$. ${ }^{* *} \mathrm{P}<0.01$. ${ }^{* * *} \mathrm{P}<0.001$. Number associated with bar graphs indicates number of mice tested. 
months of age [16]. Therefore, we wondered whether the observed affective and memory behavior impairments in $B u b R 1^{\mathrm{H} / \mathrm{H}}$ mice could be due to confounding deficits in motor function. To rule out these possibilities, we generated shRNA-BubR1 LV constructs [15] to selectively knockdown endogenous BubR1 levels within adult WT mouse DG in a non-cell autonomous manner (Fig. 2A, B). As shown in Fig. 2C, we confirmed that BubR1 knockdown significantly impairs adult hippocampal neurogenesis, as evidenced by a significant reduction in the number of EdU ${ }^{+}$cells expressed by shRNA-BubR1-LV constructs (B\#1 and B\#2) in comparison to shRNA-control. Our results are indicative of impaired neural progenitor proliferation in the hippocampal DG by deficits of BubR1. Notably, the neurogenic dysfunction denotes a consistent effect that we previously observed in cell autonomous effect of BubR1 [15]. Moreover, utilizing a GFP-retroviral approach (Fig. 2D, E), we revealed that shRNA-BubR1-LV mediated knockdown in the mouse hippocampus decreased dendrite length of newborn neurons (Fig. 2F) in comparison to shRNA-control-LV.

We next investigated whether shRNA-BubR1-LV mediated DG-specific knockdown was sufficient to potentiate memory and affective behavior deficits. Four weeks after LV injection, mice were underwent anxiety, depressive-like, and memory tests as described above. As shown in Fig. 3A, we confirmed wide-spread LV-mediated transgene expression across the septal-temporal axis of the DG. Similar to $B u b R 1^{\mathrm{H} / \mathrm{H}}$ mice, hippo-

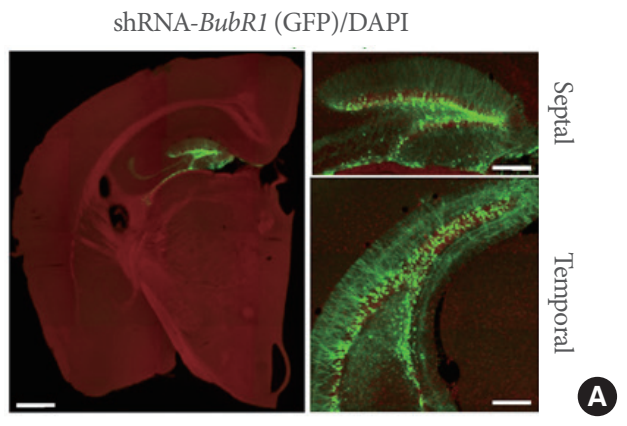

LD

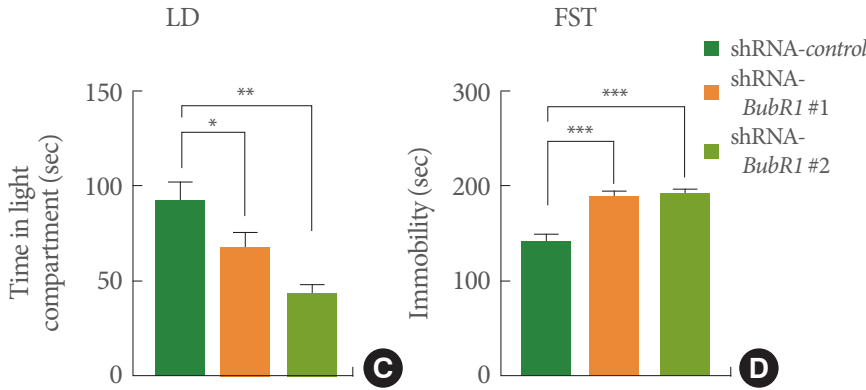

EPM

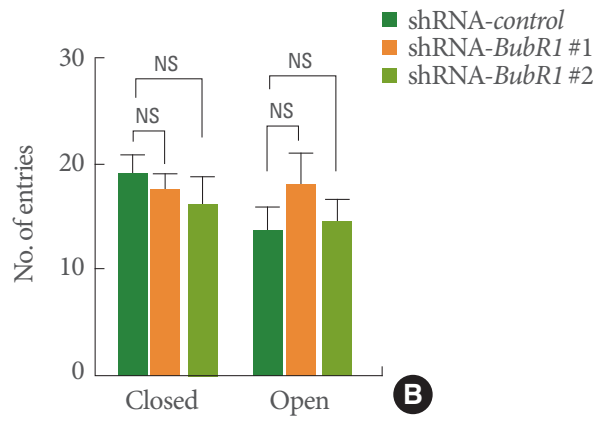

NOR
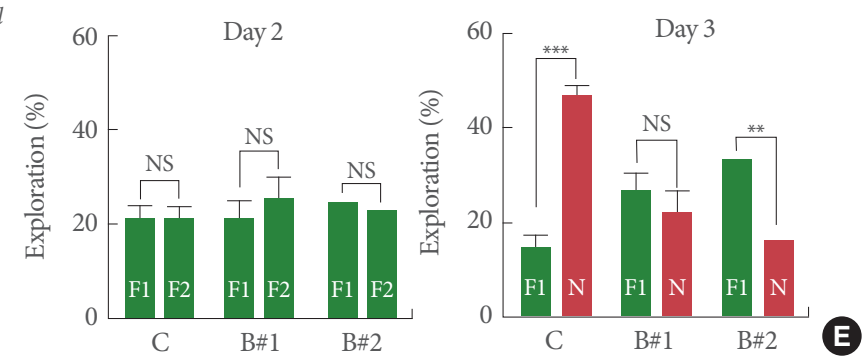

Fig. 3. Dentate gyrus (DG)-specific benzimidazole-related 1 (BubR1) knockdown in mouse hippocampus show increased anxiety, depressive-like behaviors and impaired memory function. (A) Representative images of lentiviral infected DG section. Note the wide spread of lentiviral-mediated transgene expression across the septo-temporal axis of the whole DG. Left: an enlarged view (scale bar; $1 \mathrm{~mm}$ ). Right: a high-magnification view (scale bar; $200 \mu \mathrm{m})$. (B) Elevated plus maze (EPM) test: Left is a summary of time spent in close, center and open arms. Right is a summary of number of entries into open or closed arm. Similar to BubR $1^{\mathrm{H} / \mathrm{H}}$ mice, BubR1 knockdown mice spend an increased amount of time in the closed arms and a decreased in the open arms relative to wild-type mice, indicating an increased anxiety in $B u b R 1^{\mathrm{H} / \mathrm{H}}$ mice. (C) Light-dark (LD) test: A summary of time spent in light chamber. (D) Forced swimming test (FST): Both shRNA-BubR1 \#1 and \#2 knockdown mice display increased immobility time. (E) Novel objective recognition (NOR) test: (D) Diagram of NOR test. (E) In the NOR familiarization phase on day 2, all 3 groups of mice showed no differences in the percentage of distance exploring the 2 familiar objects, indicating a lack of location preference. However, in the test phase on day 3, both shRNA-BubR1 \#1 and \#2 knockdown mice spent less time exploring the novel object, relative to BubR1 control mice, suggesting BubR1 knockdown mice show memory dysfunction. All values represent mean \pm standard error of the mean $(\mathrm{n}=10 \mathrm{mice}$ each group). F1, F2, familiar objects; N, novel object; NS, nonsignificant. 1-way analysis of variance with Bonferroni post hoc test for EPM, LD, and FST; Student t-test for NOR. ${ }^{*} \mathrm{P}<0.05 .{ }^{* *} \mathrm{P}<0.01$. ${ }^{* * *} \mathrm{P}<0.001$. 
campal DG specific knockdown of BubR1 led to higher levels of anxiety in the EPM (Fig. 3B) and LD test (Fig. 3C), increased depressive-like behavior in the FST test (Fig. 3D), and impaired memory function in NOR test (Fig. 3E). Taken together, these results indicate the critical DG-specific role of BubR1 in regulation of emotional and memory-related function.

\section{DISCUSSION}

In both humans and mice, age-related cognitive deficits are closely linked to pathology in the hippocampus, a brain area critical for memory and mood regulation [24]. The exact mechanisms mediating this decline remain unknown. In this report, we utilized a premature aging mouse model that expresses low levels of BubR1 (BubR1 $1^{\mathrm{H} / \mathrm{H}}$ mice) as well as shRNA mediated DG-specific BubR1 knockdown, to demonstrate that BubR1 insufficiency causes deficits in hippocampal-dependent memory function, increased anxiety- and depressive-like behaviors. Together with our previous studies showing that reduced levels of BubR1 impairs neurogenesis [15] and myelination [16], the results of this current study suggest that declines in BubR1 levels in conjunction with advanced aging, may be a crucial contributor to age-related hippocampal dysfunction.

We previously found that BubR1 mRNA expression is dramatically reduced in an age-dependent manner, as aging incrementally proceeds. Moreover, BubR1 insufficiency causes impairments in multiple phases of adult hippocampal neurogenesis from neural progenitor proliferation to neuronal maturation [15]. The findings in the current study extend the established function of BubR1 in age-related neuropathology to cognitive aging. Previous studies demonstrate that both humans and mice undergo declines in cognitive performance with age [24]. Additionally, adult neural progenitor proliferation and hippocampal neurogenesis dramatically decreases with age [25]. Interestingly, locomotor impairments and increased anxiety have been detected via the EPM [26], and impaired hippocampal dependent memory behaviors by the Morris water maze in aged mice [27-29], similar to what we have seen in $B u b R 1^{\mathrm{H} / H}$ mice. While the underlying mechanisms of how aging leads to hippocampal pathology are still unclear, this current study in conjunction with our previous work, demonstrate that BubR1 levels are dramatically reduced in the mouse brain in an agedependent manner [15]. Further, BubR1 $1^{\mathrm{H} / \mathrm{H}}$ mice show accelerated age-dependent impairments in neural progenitor proliferation, and impairs neuronal maturation [15]. Therefore, our re- sults suggest that BubR1 insufficiency causes premature hippocampal aging, which is consistent with previous reports stating that $B u b R 1^{\mathrm{H} / \mathrm{H}}$ mice develop accelerated aging-related pathologies in other tissues [3]. Furthermore, our results strengthen these observations, as we show that age-related BubR1 decline in the hippocampus contributes to cognitive aging.

BubR1 is commonly known as a critical mitotic checkpoint kinase, and its function is required to prevent premature anaphase initiation through inhibition of the anaphase promoting complex. In humans, mutations of BubR1 have been directly associated with aberrant neurodevelopmental traits such as microcephaly and ciliopathy, both of which are known to be associated with intellectual disability and cognitive deficits in MVA syndrome [9]. In addition, reduced levels of BubR1 are known to cause dysfunctional ciliogenesis, which leads to defects in cerebellar development [14]. Recent studies have shown that knockdown of BubR1 during embryonic neural development leads to impaired progenitor cell division and thus defects in cortical neurogenesis [30]. Taking clinical and preclinical evidence into consideration, it is highly likely that BubR1 is critical towards maintaining proper neural developmental function. Importantly, adequate presence and activation of BubR1 may be of significant physiological importance to ensure normal hippocampal function.

In conclusion, the present study shows BubR1 is essential to maintaining behavioral function and proper adult neurogenesis. A large body of evidence suggests that aging and age-related defects in hippocampal neurogenesis contributes to cognitive decline [31]. Therefore our identification of BubR1 as a critical regulator of age-related hippocampal pathology raises the possibility that BubR1 may be a key player in brain aging.

\section{CONFLICT OF INTEREST}

No potential conflict of interest relevant to this article was reported.

\section{ACKNOWLEDGEMENTS}

We would like to thank Dr. Jan M. van Deursen for providing $B u b R 1^{H / H}$ mice, and Dr. Chanil Choi for help with generating shRNA-BubR1 LV constructs. 


\section{AUTHOR CONTRIBUTION STATEMENT}

- Full access to all the data in the study and takes responsibility for the integrity of the data and the accuracy of the data analysis: $C H C, Z Y, K H Y, A O, M H J$

- Study concept and design: $\mathrm{CHC}, \mathrm{MHJ}$

- Acquisition of data: $\mathrm{CHC}, \mathrm{ZY}, \mathrm{KHY}$

- Drafting of the manuscript: $\mathrm{CHC}, \mathrm{MHJ}$

- Critical revision of the manuscript for important intellectual content: $A O, M H J$

- Obtained funding: $M H J$

- Administrative, technical, or material support: $M H J$

- Study supervision: $M H J$

\section{REFERENCES}

1. Barnes JN. Exercise, cognitive function, and aging. Adv Physiol Educ 2015;39:55-62.

2. Raftery AE, Li N, Ševčíková H, Gerland P, Heilig GK. Bayesian probabilistic population projections for all countries. Proc Natl Acad Sci U S A 2012;109:13915-21.

3. Baker DJ, Jeganathan KB, Cameron JD, Thompson M, Juneja S, Kopecka A, et al. BubR1 insufficiency causes early onset of agingassociated phenotypes and infertility in mice. Nat Genet 2004;36: 744-9.

4. Baker DJ, Dawlaty MM, Wijshake T, Jeganathan KB, Malureanu L, van Ree JH, et al. Increased expression of BubR1 protects against aneuploidy and cancer and extends healthy lifespan. Nat Cell Biol 2013;15:96-102.

5. Bolanos-Garcia VM, Blundell TL. BUB1 and BUBR1: multifaceted kinases of the cell cycle. Trends Biochem Sci 2011;36:141-50.

6. Hanks S, Coleman K, Reid S, Plaja A, Firth H, Fitzpatrick D, et al. Constitutional aneuploidy and cancer predisposition caused by biallelic mutations in BUB1B. Nat Genet 2004;36:1159-61.

7. Jacquemont S, Bocéno M, Rival JM, Méchinaud F, David A. High risk of malignancy in mosaic variegated aneuploidy syndrome. Am J Med Genet 2002;109:17-21.

8. Lane AH, Aijaz N, Galvin-Parton P, Lanman J, Mangano R, Wilson TA. Mosaic variegated aneuploidy with growth hormone deficiency and congenital heart defects. Am J Med Genet 2002;110:273-7.

9. Garcia-Castillo H, Vasquez-Velasquez AI, Rivera H, Barros-Nunez P. Clinical and genetic heterogeneity in patients with mosaic variegated aneuploidy: delineation of clinical subtypes. Am J Med Genet A 2008;146A:1687-95.

10. Bohers E, Sarafan-Vasseur N, Drouet A, Paresy M, Latouche JB,
Flaman JM, et al. Gradual reduction of BUBR1 protein levels results in premature sister-chromatid separation then in aneuploidy. Hum Genet 2008;124:473-8.

11. Suijkerbuijk SJ, van Osch MH, Bos FL, Hanks S, Rahman N, Kops GJ. Molecular causes for BUBR1 dysfunction in the human cancer predisposition syndrome mosaic variegated aneuploidy. Cancer Res 2010;70:4891-900.

12. Matsuura S, Matsumoto Y, Morishima K, Izumi H, Matsumoto H, Ito E, et al. Monoallelic BUB1B mutations and defective mitoticspindle checkpoint in seven families with premature chromatid separation (PCS) syndrome. Am J Med Genet A 2006;140:358-67.

13. Warburton D, Anyane-Yeboa K, Taterka P, Yu CY, Olsen D. Mosaic variegated aneuploidy with microcephaly: a new human mitotic mutant? Ann Genet 1991;34:287-92.

14. Miyamoto T, Porazinski S, Wang H, Borovina A, Ciruna B, Shimizu A, et al. Insufficiency of BUBR1, a mitotic spindle checkpoint regulator, causes impaired ciliogenesis in vertebrates. Hum Mol Genet 2011;20:2058-70.

15. Yang Z, Jun H, Choi CI, Yoo KH, Cho CH, Hussaini SM, et al. Agerelated decline in BubR1 impairs adult hippocampal neurogenesis. Aging Cell 2017;16:598-601.

16. Choi CI, Yoo KH, Hussaini SM, Jeon BT, Welby J, Gan H, et al. The progeroid gene BubR1 regulates axon myelination and motor function. Aging (Albany NY) 2016;8:2667-88.

17. Walf AA, Frye CA. The use of the elevated plus maze as an assay of anxiety-related behavior in rodents. Nat Protoc 2007;2:322-8.

18. Jang MH, Kitabatake Y, Kang E, Jun H, Pletnikov MV, Christian KM, et al. Secreted frizzled-related protein 3 (sFRP3) regulates antidepressant responses in mice and humans. Mol Psychiatry 2013;18:957-8.

19. Hascoet M, Bourin M. A new approach to the light/dark test procedure in mice. Pharmacol Biochem Behav 1998;60:645-53.

20. Leger M, Quiedeville A, Bouet V, Haelewyn B, Boulouard M, Schumann-Bard P, et al. Object recognition test in mice. Nat Protoc 2013; 8:2531-7.

21. Ge S, Goh EL, Sailor KA, Kitabatake Y, Ming GL, Song H. GABA regulates synaptic integration of newly generated neurons in the adult brain. Nature 2006;439:589-93.

22. Jang MH, Bonaguidi MA, Kitabatake Y, Sun J, Song J, Kang E, et al. Secreted frizzled-related protein 3 regulates activity-dependent adult hippocampal neurogenesis. Cell Stem Cell 2013;12:215-23.

23. Cohen SJ, Stackman RW Jr. Assessing rodent hippocampal involvement in the novel object recognition task. A review. Behav Brain Res 2015;285:105-17.

24. Bettio LE, Rajendran L, Gil-Mohapel J. The effects of aging in the hippocampus and cognitive decline. Neurosci Biobehav Rev 2017; 
79:66-86.

25. Ernst A, Frisén J. Adult neurogenesis in humans- common and unique traits in mammals. PLoS Biology 2015;13:e1002045.

26. Fahlström A, Yu Q, Ulfhake B. Behavioral changes in aging female C57BL/6 mice. Neurobiol Aging 2011;32:1868-80.

27. Barreto G, Huang TT, Giffard RG. Age-related defects in sensorimotor activity, spatial learning, and memory in C57BL/6 mice. J Neurosurg Anesthesiol 2010;22:214-9.

28. Villeda SA, Plambeck KE, Middeldorp J, Castellano JM, Mosher KI, Luo J, et al. Young blood reverses age-related impairments in cognitive function and synaptic plasticity in mice. Nat Med 2014;20:659-63.

29. de Fiebre NC, Sumien N, Forster MJ, de Fiebre CM. Spatial learning and psychomotor performance of C57BL/6 mice: age sensitivity and reliability of individual differences. Age 2006;28:235-53.

30. Guo J, Higginbotham H, Li J, Nichols J, Hirt J, Ghukasyan V, et al. Developmental disruptions underlying brain abnormalities in ciliopathies. Nat Commun 2015;6:7857.

31. Lazarov O, Mattson MP, Peterson DA, Pimplikar SW, van Praag H. When neurogenesis encounters aging and disease. Trends Neurosci 2010;33:569-79. 